\title{
Cardiovascular and Interventional Radiological Society of Europe Commentary on the Treatment of Chronic Cerebrospinal Venous Insufficiency
}

\author{
J. A. Reekers $\cdot$ M. J. Lee $\cdot$ A. M. Belli • \\ F. Barkhof
}

Received: 1 November 2010/Accepted: 3 November 2010/Published online: 7 December 2010

(C) The Author(s) 2010. This article is published with open access at Springerlink.com

\begin{abstract}
Chronic cerebrospinal venous insufficiency (CCSVI) is a putative new theory that has been suggested by some to have a direct causative relation with the symptomatology associated with multiple sclerosis (MS) [1]. The core foundation of this theory is that there is abnormal venous drainage from the brain due to outflow obstruction in the draining jugular vein and/or azygos veins. This abnormal venous drainage, which is characterised by special ultrasound criteria, called the "venous hemodynamic insufficiency severity score" (VHISS), is said to cause intracerebral flow disturbance or outflow problems that lead to periventricular deposits [2]. In the CCSVI theory, these deposits have a great similarity to the iron deposits seen around the veins in the legs in patients with chronic deep vein thrombosis. Zamboni, who first described this new theory, has promoted balloon dilatation to treat the outflow problems, thereby curing CCSVI and by the same token alleviating MS complaints. However, this theory does not fit into the existing bulk of scientific data concerning the pathophysiology of MS. In contrast,
\end{abstract}

\footnotetext{
J. A. Reekers $(\bowtie)$

Department of Radiology, AMC, University of Amsterdam,

Amsterdam, The Netherlands

e-mail: j.a.reekers@amc.uva.nl
}

M. J. Lee

Department of Academic Radiology,

Beaumont Hospital, Dublin, Ireland

\author{
A. M. Belli \\ Department of Radiology, St. George's Hospital, \\ London, England \\ F. Barkhof \\ Department of Radiology, MS Center Amsterdam, \\ VU University Medical Center, Amsterdam, \\ The Netherlands
}

there is increasing worldwide acceptance of CCSVI and the associated balloon dilatation treatment, even though there is no supporting scientific evidence. Furthermore, most of the information we have comes from one source only. The treatment is called "liberation treatment," and the results of the treatment can be watched on YouTube. There are well-documented testimonies by MS patients who have gained improvement in their personal quality of life (QOL) after treatment. However, there are no data available from patients who underwent unsuccessful treatments with which to obtain a more balanced view. The current forum for the reporting of success in treating CCSVI and thus MS seems to be the Internet. At the CIRCE office and the MS Centre in Amsterdam, we receive approximately 10 to 20 inquiries a month about this treatment. In addition, many interventional radiologists, who are directly approached by MS patients, contact the Cardiovascular and Interventional Radiological Society of Europe (CIRSE) for advice. Worldwide, several centres are actively promoting and performing balloon dilatation, with or without stenting, for CCSVI. Thus far, no trial data are available, and there is currently no randomized controlled trial (RCT) in progress Therefore, the basis for this new treatment rests on anecdotal evidence and successful testimonies by patients on the Internet. CIRSE believes that this is not a sound basis on which to offer a new treatment, which could have possible procedure-related complications, to an often desperate patient population.

\section{Introduction}

CIRSE believes that the CCSVI theory in its current form has a number of inconsistencies. The core of this new theory is the CCSVI syndrome, i.e., abnormal venous 
drainage from the brain. However, venous drainage from the head has an impressive anatomic variation, which has not been well catalogued in most textbooks. In addition, valves can be present at a variety of sites in the head and neck veins. Indeed, interventional radiologists who practise parathyroid sampling know about this huge variety in venous anatomy. In addition to the huge variation in normal venous anatomy, the jugular veins have some natural narrowing at two sites, and the azygos vein, so prominent in CCSVI theory, does not drain the brain at all. The azygous vein obviously can drain the spinal cord, but not solely, because many intercostal venous collateral veins fulfil the same function.

In addition, the fact that this imaging is performed with the patient in the supine position has a great influence on blood flow and image interpretation. There recently have been randomised studies comparing so-called "venous stenoses" in patients with and without MS [3, 4]. Both studies showed that there was no difference in the prevalence of venous stenoses between the two groups. This seems to be a strong argument against the existence of CCSVI. However, those physicians performing CCSVI treatment point out that these studies were not performed according to the VHISS criteria. The other part of the theory is that the venous outflow obstruction must be treated. However, if there is a real haemodynamic venous outflow obstruction, there should also be a pressure gradient, and this gradient should disappear after successful balloon dilatation. It has been shown, and confirmed by those who perform balloon dilatation for CCSVI, that over the so-called "stenosis" there is never a measurable pressure gradient. Again, those who perform this treatment will say that it is not the pressure gradient but the change in outflow pattern caused by this stenosis that is the pathologic entity.

What remains is the issue of anecdotal successful treatments. Undoubtedly, some patients obtain symptom relief after treatment for CCSVI, but this could easily be just a placebo effect. Many treatment successes in medicine are based on or helped by a placebo effect. In itself, there is nothing wrong with the placebo effect as long as we recognize that this is at play. It is also known that the more invasive the treatment and the more the treating physician believes in the treatment, the stronger the placebo effect is. Furthermore, MS can affect emotional and labile responses and is characterized by spontaneous relapses and remissions. This makes the gathering of scientific evidence to support CCSVI theory difficult in anything other than an RCT.

What we now have is the dilemma of a new treatment being promoted and performed by some early pioneers, with the popular press trumpeting its success and thus decreasing the chances of performing a properly conducted trial because it is considered unethical not to offer patients this new and promising treatment. The primary task of physicians is primum non nocere or not doing harm. We believe that harm can be caused by offering treatments without any scientific proof of efficacy as well as the more usual forms of medical harm. Pseudo-arguments such as "There is nothing else" or "Do you know how much they pay for medication that does not work?" are not valid and scientific arguments.

Confronted with these contradictions and lack of evidence for CCSVI treatment, some pioneers have taken to calling their work on MS patients a "phase 1 study." However, a phase 1 study without a protocol, that has not been approved by a medical ethical committee, that does not require informed consent, and that does not have safety committee oversight does not qualify! CIRSE believes that only properly conducted trials with significant scientific rigor can solve this dilemma. We at CIRCE believe that a small prospective randomised trial, with a sham arm, is required. A trial monitored by an independent society, such as CIRSE and/or the European Society of Neurology, would be ideal.

As doctors and interventional radiologists, we certainly hope that all of the anecdotal reports detailing improvements in QOL will prove to be true and that patients will benefit from this new treatment. Interventional radiologists have a long history of introducing pioneering treatments during the last 30 years that have proven to be of enormous benefit to patients. Recently new treatments, such as fibroid embolization, vertebroplasty, and carotid stenting, have all been interrogated by randomized trials. We believe that CCSVI treatment should be evaluated in the same manner. Furthermore, we believe that until real scientific data are available for CCSVI and balloon dilatation, this treatment should not be offered to MS patients outside of a welldesigned clinical trial.

Open Access This article is distributed under the terms of the Creative Commons Attribution Noncommercial License which permits any noncommercial use, distribution, and reproduction in any medium, provided the original author(s) and source are credited.

\section{References}

1. Zamboni P (2006) The big idea: iron-dependent inflammation in venous disease and proposed parallels in multiple sclerosis. J R Soc Med 99:589-593

2. Zamboni P, Menegatti E, Weinstock-Guttman B, Schirda C et al (2009) The severity of chronic cerebrospinal venous insufficiency in patients with multiple sclerosis is related to altered cerebrospinal fluid dynamics. Funct Neurol 24:133-138

3. Krogias C, Schröder A, Wiendl H, Hohlfeld R, Gold R (2010) Chronic cerebrospinal venous insufficiency" and multiple sclerosis: critical analysis and first observation in an unselected cohort of MS patients. Nervenarzt 81:740-746

4. Doepp F, Paul F, Valdueza JM, Schmierer K, Schreiber SJ (2010) No cerebrocervical venous congestion in patients with multiple sclerosis. Ann Neurol 68:173-183 